\title{
Breast Cancer Screening Practice and Associated Factors in Menopausal and Postmenopausal Women
}

\author{
Florence Mei Fung Wong, Winnie Lai Sheung Cheng \\ School of Nursing, Tung Wah College, Kowloon, Hong Kong SAR, China
}

Objectives: This study examined breast cancer screening (BCS) practices and its associated factors among menopausal and postmenopausal women.

Methods: A cross-sectional design was conducted using convenience sampling at a community center. The modified Chinese BCS Belief questionnaire was used to understand BCS practice.

Results: A total of 144 eligible women presented better knowledge and perceptions about breast cancer (mean, 11.46; standard deviation [SD], 3.65) and barriers to achieving mammographic screening (mean, 14.75; SD, 3.70). Participants aged $\geq 50$ years had a negative association with the attitudes towards general health check-ups $(B=-1.304$, standard error $[S E]=0.65, P=0.046)$. In this context, having regular physical exercise had a positive association with attitudes towards general health check ups $(B=1.458$, $\mathrm{SE}=0.06, P=0.017)$, and knowledge and perceptions about breast cancer $(B=1.068, \mathrm{SE}=0.62, P=0.086)$. Being employed had a positive associated with barriers to achieving mammographic screening $(B=1.823, S E=0.51, P<0.001)$.

Conclusions: The women had better knowledge and perception about breast cancer and fewer barriers to mammographic screening. However, attitudes towards general health check-ups were relatively poor. It is noteworthy that women who aged $\geq 50$ years and had insufficient physical exercise had poor attitudes. Those who were employed had fewer barriers, and those who had regular physical exercise had better knowledge on the benefits of the BCS practice. Importantly, women who aged $\geq 50$ years and lack physical exercise need education about breast cancer to increase their awareness of breast wellness. (J Menopausal Med 2019;25:4148)

Key Words: Breast neoplasms · Menopause · Postmenopause · Women's health

\section{Introduction}

Breast cancer has been the cause of high mortality rate worldwide and in Hong Kong. ${ }^{1-3}$ In Hong Kong, breast cancer has become the third killer, surpassing lung cancer and colorectal cancers since the 1990s, ${ }^{4,5}$ Breast cancer tends to occur in younger ages from 40 years old and the most prevalent risk group of Hong Kong Chinese women is from aged 50 years. ${ }^{6,7}$
Breast cancer can be preventable as long as the risks of breast cancer are removed or controlled if breast cancer screening (BCS) is commenced at an early stage. ${ }^{7-9}$ BCS practice consists of breast self-examination (BSE), clinical breast examination, and mammogram for early detection measure. ${ }^{10-12}$ Although BSE is the simplest and most convenient method to detect breast abnormalities, such as lumps and discharge from nipple, it is uncommon in Asian women. ${ }^{7,8,13-15}$ To effectively reduce the mortality of breast cancer,

Received: September 27, 2018 Revised: January 1, 2019 Accepted: Febuary 12, 2019

Address for Correspondence: Florence Mei Fung Wong, School of Nursing, Tung Wah College, 31 Wylie Road, Homantin, Kowloon, Special Administrative Region, Hong Kong SAR, China

Tel: +852-34686838, Fax: +852-27821566, E-mail: florencewong@twc.edu.hk

ORCID: https://orcid.org/0000-0002-9544-2607 
it is important to identify factors associated with health beliefs towards BCS.

The risk factors of developing breast cancer can be copious. Evidence showed that lacking of physical exercise, alcohol consumption, obesity, no childbirth or breastfeeding experience, and receiving hormonal replacement were closely associated with breast cancer. ${ }^{4}$ Misconception and inadequate knowledge can explain the indifference and disregard to the importance of BCS among Chinese women. ${ }^{15}$ Therefore, this study aimed to examine attitudes, perceptions and barriers towards BCS practice and identify factors associated with BCS practice among menopausal and postmenopausal Chinese women. The findings can be added in the existing knowledge to increase awareness of breast wellness contributing to promote BCS practice.

To facilitate the understanding of study purpose, a theoretical framework, the Health Belief Model (HBM) proposed by Rosenstock ${ }^{16}$ was adopted to address our study purpose. This HBM is a conceptual framework commonly used to identify factors associated with individual beliefs that influence behaviors. Rosenstock's model has been commonly used to explore attitudes, perception and barriers towards BCS. ${ }^{7,9,17-20}$ It consists of four concepts: (a) perceived personal susceptibility to a disease, (b) perceived seriousness of the harm of a disease, (c) perceived benefits of taking a health action, and (d) perceived barriers to taking a health action.

\section{Materials and Methods}

\section{Design and sampling}

A descriptive cross-sectional study was conducted. A convenience sampling of menopausal and postmenopausal women was recruited from a community center in an urban district in Hong Kong. Sample size calculation was performed using PASS 11 (NCSS, LLC., Kaysville, UT, USA). A sample size of 118 subjects was estimated to provide the study with a power of $80 \%$ in detecting a factor with a medium effect size of Cohen's $\mathrm{f}^{2}=0.15$ at $5 \%$ type I error rate using multivariable regression with up to 10 candidate explanatory variables. ${ }^{21}$ The inclusion criteria were women aged 40 years old or above without any mental health problems and they were able to communicate in Chinese.

\section{Outcome variables}

The original Chinese Breast Cancer Screening Belief (CBCSB) was developed with good validity and reliability by Kwok et al. ${ }^{22}$ The CBCSB was used to measure Chinese women's perception and knowledge, attitudes about breast cancer and barriers to mammographic screening practice. ${ }^{23}$ The modified CBCSB was designed to be more suitable to Chinese women with consideration of their living environment and cultural background. The modified CBCSB consists of 11 items with three domains: attitudes towards general health check-ups (4 items); knowledge and perceptions about breast cancer (4 items); and barriers to mammographic screening (3 items). All items were rated using a 5-point Likert scale. The lower score indicates the lowest attitude, least knowledge or greatest barrier and vice versa. The total scores of each domain were calculated following the formula (total score - minimum total)/(maximum total - minimum total). The Cronbach's alpha of three domains (attitudes towards general health check-ups, knowledge and perceptions about breast cancer, and barriers to mammographic screening) and the overall modified CBCSB were 0.70, 0.75, 0.80, and 0.76 respectively in this study indicating that they all were good reliability.

\section{Data collection}

Data were collected using one set of questionnaires consisting of one demographic form and the CBCSB through structured researcher-administered face-to-face interviews. They were required to sign an informed consent after the study purpose was explained. The set of questionnaires included a questionnaire to gather demographic information and a modified CBCSB questionnaire. The questionnaires were completed through face-to-face interviews. The interview took about 20 minutes.

\section{Data analysis}

Data were analyzed by IBM SPSS Statistics ver. 20.0 (IBM Corp., Armonk, NY, USA). Descriptive statistics was performed to summarize and present participants' characteristics and outcome variables (attitudes towards general health check-ups, knowledge and perceptions about breast cancer, and barriers to mammographic screening). Univariate analyses between the independent and dependent vari- 
ables were assessed using Pearson's correlation coefficient, independent-samples $t$ test, or one-way ANOVA, depending on the level of measurement of the independent variables, in identifying candidate independent variables for multivariable regression. For each subscale of BCS practice, independent variables with $P$ values less than 0.25 in univariate analyses were selected as candidate independent variables for multivariable regression analysis to delineate associated factors independently. ${ }^{24}$ All statistical tests involved were twosided and the type I error rate (level of significance) was set at 0.05. Pearson's correlations were used to determine the relationships between variables. All statistical analysis was determined with two-sided and the type I error rate (level of significance) at 0.05 .

\section{Ethical considerations}

Approvals were obtained from the Research Ethics Committee of a self-finance educational institute (no. 2015-00-55 R150401). Eligible women who agreed to participate in the study were requested to sign a written consent and their personal information was kept confidential and anonymous.

\section{Results}

\section{Demographic characteristics, health practice, and knowledge about breast cancer}

A total of 144 female participants were recruited. None of them was diagnosed with breast cancer. Most of them were aged 50 years or older (69.4\%). More than 80\% $(n=122)$ of the female participants were married. Of the 144 participants, 102 (70.8\%) were educated with attained secondary or higher education. However, 60.4\% ( $\mathrm{n}=87$ ) were unemployed and about half of them were homemakers or retired. Regarding their lifestyles, 93.1\% ( $\mathrm{n}=134)$ were non-smokers and $95.8 \%(\mathrm{n}=138)$ were non-drinkers. However, about half of them $(\sim 56 \%)$ participated in regular physical exercise that is defined as a 30-minute aerobic exercise, such as brisk walking, swimming, and cycling, for five times a week. Ninety-eight participants (68.1\%) expressed they were worried about having breast cancer after menopause. Regarding knowledge about breast cancer that is related to the subjects' general understanding about breast cancer, about most of
Table 1. Sample characteristics, health practice and knowledge about breast cancer

\begin{tabular}{|c|c|}
\hline Variable & Data \\
\hline Total & $144(100)$ \\
\hline \multicolumn{2}{|l|}{ Age (y) } \\
\hline$<50$ & $44(30.6)$ \\
\hline $50-59$ & $65(45.1)$ \\
\hline $60-79$ & $31(21.5)$ \\
\hline$\geq 80$ & $4(2.8)$ \\
\hline \multicolumn{2}{|l|}{ Marital status } \\
\hline Single & $24(16.7)$ \\
\hline Married & $120(83.3)$ \\
\hline \multicolumn{2}{|l|}{ Educational level } \\
\hline Primary school or below & $42(29.2)$ \\
\hline Secondary school & $75(52.1)$ \\
\hline Tertiary or above & $27(18.8)$ \\
\hline \multicolumn{2}{|l|}{ Occupation } \\
\hline Unemployed or retired & $87(60.4)$ \\
\hline Employed & $57(39.6)$ \\
\hline \multicolumn{2}{|l|}{ Drinking habit } \\
\hline No & $138(95.8)$ \\
\hline Yes & $6(4.2)$ \\
\hline \multicolumn{2}{|l|}{ Smoking habit } \\
\hline No & $134(93.1)$ \\
\hline Yes & $10(6.9)$ \\
\hline \multicolumn{2}{|l|}{ Regular physical exercise } \\
\hline No & $64(44.4)$ \\
\hline Yes & $80(55.6)$ \\
\hline
\end{tabular}

Had breast examination by a doctor in the past one year

$$
\begin{array}{ll}
\text { No } & 64(44.4) \\
\text { Yes } & 80(55.6)
\end{array}
$$

Knowledge about breast cancer

$\begin{array}{ll}\text { No } & 53(36.8) \\ \text { Yes } & 25(17.4) \\ \text { Average } & 66(45.8)\end{array}$

Worry about having breast cancer after menopause

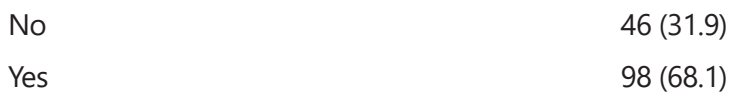

Want to receive information about breast cancer

$\begin{array}{ll}\text { No } & 46(31.9) \\ \text { Yes } & 98(68.1)\end{array}$

The data is presented as number (\%). 
the participants $(82.6 \%)$ reported that they had insufficient to average knowledge about breast cancer. More than half ( $\sim 68 \%)$ hoped to receive more information about it. About $56 \%$ of the participants had had a breast examination by a doctor in the past year. The sample characteristics, health practice and knowledge about breast cancer are summarized in Table 1.

\section{Attitudes towards general health check-ups, knowledge and perceptions about breast cancer, and barriers to mammographic screening}

Figure 1 shows the responses to each item of the CBCSB. The results showed that most of the women were not willing to have medical consultation unless they were sick. Regarding breast cancer, most of the women had confidence in the cure of breast cancer. More than $60 \%$ of women had positive perspectives towards having breast mammogram. Table 2 illustrates the analytic results of three domains of BCS beliefs. The results showed that knowledge and perceptions about breast cancer (mean, 14.75; standard deviation [SD], 3.70) and barriers to mammographic screening (mean, 11.62; SD, 3.11) were better than attitudes towards general health check-ups (mean, 11.46; SD, 3.65). The total scores of attitudes towards general health check-ups, knowledge and perceptions about breast cancer and barriers to mammographic screening were 102.88, 132.5, and 139.17, respectively.

\section{Variables associated with/among three domains of breast cancer screening beliefs}

Table 3 showed the results of univariate analysis between independent variables and three domains of breast cancer

Table 2. Analytic results of three domains of breast cancer screening beliefs

\begin{tabular}{ccc}
\hline $\begin{array}{c}\text { Domains of health beliefs on } \\
\text { breast cancer }\end{array}$ & Data & $\begin{array}{c}\text { Total } \\
\text { score* }^{*}\end{array}$ \\
\hline $\begin{array}{c}\text { Attitudes towards general } \\
\text { health check-ups }\end{array}$ & $11.46 \pm 3.65$ & 102.88 \\
$\begin{array}{c}\text { Knowledge and perceptions about } \\
\text { breast cancer }\end{array}$ & $14.75 \pm 3.70$ & 132.50 \\
$\begin{array}{c}\text { Barriers to mammographic screening } \\
\text { nen }\end{array}$ & $11.62 \pm 3.11$ & 139.17 \\
\hline
\end{tabular}

The data is presented as mean \pm standard deviation or score only. *Range, 35.75-179.75.
1. If I feel well, I don't think it is necessary to go far a health check-up

2. If I follow a healthy lifestyle such as a balanced diet and regular exercise, I don't feel it is necessary to have a regular check-up

3. I see a doctor or have my health check-up only when I have a health problem

4. If I feel healthy, I do not go to the doctor

5. Breast cancer is like a death sentence; if you get it, you will surely die from it

6. Breast cancer cannot be cured; you can only prolong the suffering

7. Even if breast cancer is detected early, there is very little a woman can do to reduce the chances of dying from it

8. If a woman is fated to get breast cancer, she will get breast cancer, there is nothing she can do to change fate

9. I'm worried that having a mammogram will hurt my breasts

10. I don't want to go for a mammogram because I would need to take off my clothes and expose my breasts

11. Having a mammogram is embarrassing

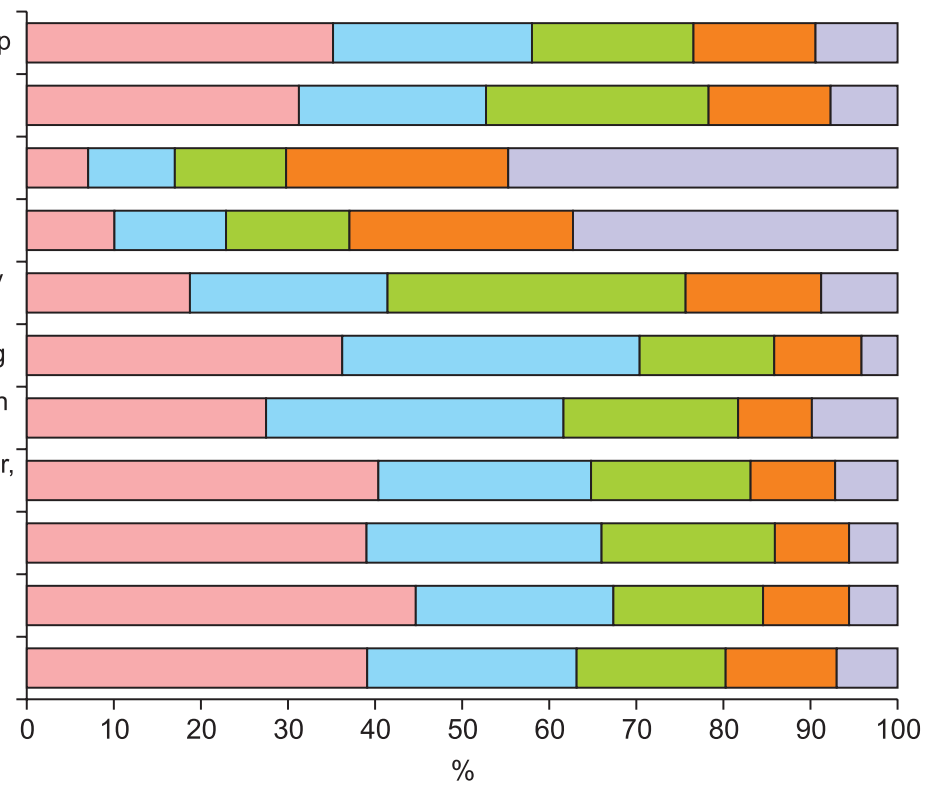

Strongly disagree

Disagree

Neutral

Strongly agree

Agree

Fig. 1. Knowledge, attitudes, and practice towards breast cancer screening practice. 
Table 3. Univariate analysis between independent variables and three domains of breast cancer screening practice

\begin{tabular}{|c|c|c|c|c|c|c|}
\hline \multirow[t]{2}{*}{ Variable } & \multicolumn{2}{|c|}{$\begin{array}{l}\text { Attitudes towards general } \\
\text { health check-ups }\end{array}$} & \multicolumn{2}{|c|}{$\begin{array}{l}\text { Knowledge and perceptions } \\
\text { about breast cancer }\end{array}$} & \multicolumn{2}{|c|}{$\begin{array}{l}\text { Barriers to mammographic } \\
\text { screening }\end{array}$} \\
\hline & Pearson & $P$ value & Pearson & $P$ value & Pearson & $P$ value \\
\hline Age & -0.144 & $0.084^{*}$ & 0.087 & 0.301 & -0.057 & 0.494 \\
\hline Marital status & 0.005 & 0.951 & 0.014 & 0.869 & -0.073 & 0.384 \\
\hline Education & 0.102 & $0.224^{*}$ & -0.047 & 0.578 & 0.099 & $0.240^{*}$ \\
\hline Occupation & 0.093 & 0.267 & 0.097 & 0.250 & 0.288 & $<0.001^{\ddagger}$ \\
\hline Drinking & -0.103 & $0.221^{*}$ & 0.051 & 0.546 & 0.071 & 0.401 \\
\hline Smoking & 0.056 & 0.508 & -0.079 & 0.345 & -0.072 & 0.391 \\
\hline Regular physical exercise & 0.182 & $0.029^{+}$ & -0.148 & $0.077^{*}$ & -0.007 & 0.938 \\
\hline
\end{tabular}

${ }^{\star} P<0.25,{ }^{+} P<0.05,{ }^{\ddagger} P<0.001$.

Table 4. Multivariable regression results of association between independent variables and three domains of breast cancer screening beliefs $(n=144)$

\begin{tabular}{|c|c|c|c|c|c|c|c|c|c|}
\hline \multirow{2}{*}{ Variable } & \multicolumn{3}{|c|}{$\begin{array}{l}\text { Attitudes towards general } \\
\text { health check-ups }\end{array}$} & \multicolumn{3}{|c|}{$\begin{array}{l}\text { Knowledge and perceptions } \\
\text { about breast cancer }\end{array}$} & \multicolumn{3}{|c|}{$\begin{array}{l}\text { Barriers to mammographic } \\
\text { screening }\end{array}$} \\
\hline & B & SE & $P$ & B & SE & $P$ & B & SE & $P$ \\
\hline Age, $\geq 50 y$ & -1.304 & 0.65 & $0.046^{*}$ & & & & & & \\
\hline Having occupation & & & & & & & 1.823 & 0.51 & $<0.001^{\star \star}$ \\
\hline Having regular physical exercise & 1.458 & 0.06 & $0.017^{\star}$ & 1.068 & 0.62 & 0.086 & & & \\
\hline
\end{tabular}

SE: standard error.

${ }^{*} P<0.05,{ }^{* *} P<0.001$.

screening practice conducted to identify independent variables that were potentially associated with three domains of BCS beliefs. Variables when $P<0.25$ were selected for multivariable regression. Multivariable regression identified independent variables that were associated with three domains of BCS beliefs. Younger age and having regular physical exercise were found to have negative association $(B=-1.304$, standard error $[\mathrm{SE}]=0.65, P=0.046$; and $\mathrm{B}=1.458, \mathrm{SE}=$ $0.06, P=0.017$, respectively) with attitudes towards general health check-ups $(\mathrm{R} 2=0.060, \mathrm{~F}[2,141]=4.50, P<0.001)$. Having occupation had a positive association $(\mathrm{B}=1.823, \mathrm{SE}$ $=0.51, P<0.001)$ with the barriers to BCS practice $(\mathrm{R} 2=$ $0.083, \mathrm{~F}[1,142]=12.80, P<0.001)$. Having regular physical exercise was also found to be significantly associated with knowledge about BCS practices $(\mathrm{R} 2=0.021, \mathrm{~F}[1,142]=2.99$, $P=0.086$ ). Table 4 shows the details of the multivariate re- gression results of association between independent variables and three domains of breast cancer screening beliefs.

\section{Discussion}

The study aimed to understand health practice on BCS and to identify factors associated with the health practice among menopausal and postmenopausal Chinese women. Most of the women were well-educated with secondary school levels or above ( 70\%), but they reported themselves as having poor perception about breast cancer and were worried about having breast cancer after menopause. They wanted to have more information about breast cancer. It can be explained by the fact that education about breast cancer often does not effectively reach at-risk women, ${ }^{11,13}$ leading to 
poor public awareness about breast cancer and its screening practice. As a result, most of the women $(\sim 55 \%)$ did not have regular breast examination practice or seek medical consultation.

Based on the HBM, an individual's health concern motivates behaviors to promote health or maintain well-being. ${ }^{16}$ Therefore, women who do not seek for medical consultation do not perceive susceptibility and severity of breast cancer as a threat. Therefore, it is important to enhance knowledge among at-risk women via community-based educational programs and healthcare promotion schemes on preventive measures for breast cancer to increase awareness of breast wellness and promote better breast care. ${ }^{25-28}$ Moreover, Chinese tradition can play an important role in increasing women's hesitation to have BCS practice as they are more likely to have misconception about BCS. ${ }^{27,28}$ Moreover, clinical breast cancer examination will be performed by a male physician and the women can feel embarrassed or even humiliated. ${ }^{7}$

The findings also revealed that women who were old $(\geq$ 50 years old) and did not have regular physical exercise perceive poorer attitudes towards general health check-ups. Most of the women were menopausal and post-menopausal ages ( $\geq 50$ years old); only half of the women reported to have regular 30-minutes physical exercise for five times a week. Based on the HBM, those women did not perceive susceptibility to and the severity of breast cancer as a threat. Hence, their attitudes to general health checkups were reflected. ${ }^{16,29,30}$ The at-risk women are more likely to perceive low level of breast awareness for regular BCS, probably attributed to inadequate knowledge about breast cancer and its preventive care.

Since women are usually housewives and responsible for taking care of the family, it is important to use appropriate channels to enhance their awareness and attitudes towards BCS practice. ${ }^{26,29,30}$ Some working women perceived more barriers to mammographic screening due to time constraint, heavy accountability of taking care of family, and expensive mammographic screening. ${ }^{29,30}$ Women who perform regular physical exercise pay more attention to their health. Maintaining regular physical exercise has evidenced as a resilience to reduce the risk of cancers. ${ }^{27,31,32}$ Evidence showed that such behavior can be attributed to adequate knowledge about breast cancer and its preventive measures. ${ }^{29,31-33}$

This study has some limitations. First, our results may not provide causal inferences between factors and attitudes, knowledge and barriers towards BCS practice because of the study design. Second, the study results cannot be generalized to other women in various cultures because Chinese women were selected. Therefore, a larger sample is recommended in future studies.

The BCS practice is an important detection to reduce the mortality by breast cancer. This study reported better knowledge and perception about breast cancer and fewer barriers to mammographic screening but relative poor attitudes towards general health check-ups in menopausal and postmenopausal women. The at-risk women had poor breast awareness for regular BCS. Effective and appropriate breast wellness and its preventive promotion scheme should be developed to support and increase their awareness and enhance the understanding of breast cancer in at-risk women for better breast care. Factors associated with health practice on BCS screening were identified, including age $(\geq$ 50 years old), having occupation and having regular physical exercise. Educational promotion scheme may be useful to increase awareness of breast cancer among at risk women.

\section{Acknowledgements}

We would like to express our gratitude to the women who participated in this study and the colleagues who helped organize the health talk.

\section{Conflict of Interest}

This study was supported with fund by Takeda Pharmaceuticals (Hong Kong) Limited. No potential conflict of interest relevant to this article was reported.

\section{References}

1. McCracken M, Olsen M, Chen MS Jr, Jemal A, Thun M, 
Cokkinides V, et al. Cancer incidence, mortality, and associated risk factors among Asian Americans of Chinese, Filipino, Vietnamese, Korean, and Japanese ethnicities. CA Cancer J Clin 2007; 57: 190-205.

2. Siegel RL, Miller KD, Jemal A. Cancer statistics, 2016. CA Cancer J Clin 2016; 66: 7-30.

3. World Health Organization (WHO). Breast cancer awareness month in October. Geneva: WHO, 2016. [cited by 2017 Jan 27]. Available from: http://www.who.int/cancer/events/ breast_cancer_month/en/.

4. Centre for Health Protection (CHP). Breast cancer. Kowloon: CHP, 2016. [cited by 2017 Jan 28]. Available from: http://www. chp.gov.hk/en/content/9/25/53.html.

5. Hospital Authority. Overview of 2013 Hong Kong cancer statistics. Kowloon: Hospital Authority, 2013. [cited by 2017 Jan 15]. Available from: https://www3.ha.org.hk/cancereg/ pdf/overview/Summary\%20of\%20CanStat\%202013.pdf.

6. Hospital Authority. Breast cancer. Kowloon: Hospital Authority, 2016. [cited by 2017 Jan 13]. Available from: https://www21. ha. org.hk/smartpatient/SPW/en-US/Disease-Information/Disease/?guid=bc5c075d-d161-4abc-9b1b1b1ca15d6947.

7. Parsa P, Kandiah M, Mohd Nasir MT, Hejar AR, Nor Afiah MZ. Reliability and validity of Champion's Health Belief Model Scale for breast cancer screening among Malaysian women. Singapore Med J 2008; 49: 897-903.

8. Kim JI, Oh KO, Li CY, Min HS, Chang ES, Song R. Breast cancer screening practice and health-promoting behavior among Chinese women. Asian Nurs Res (Korean Soc Nurs Sci) 2011; 5: 157-63

9. Tastan S, Iyigün E, Kılıc A, Unver V. Health beliefs concerning breast self-examination of nurses in Turkey. Asian Nurs Res (Korean Soc Nurs Sci) 2011; 5: 151-6.

10. Oeffinger KC, Fontham ET, Etzioni R, Herzig A, Michaelson JS, Shih YC, et al. Breast cancer screening for women at average risk: 2015 guideline update from the American Cancer Society. JAMA 2015; 314: 1599-614.

11. Yan YY. Breast cancer: knowledge and perceptions of Chinese women in Hong Kong. Glob J Health Sci 2009; 1: 97105.

12. Abbaszadeh A, Haghdoost AA, Taebi M, Kohan S. The relationship between women's health beliefs and their participation in screening mammography. Asian Pac J Cancer Prev 2007; 8: 471-5.

13. Wang WL, Hsu SD, Wang JH, Huang LC, Hsu WL. Survey of breast cancer mammography screening behaviors in Eastern Taiwan based on a health belief model. Kaohsiung J Med Sci 2014; 30: 422-7.

14. Gomez SL, Tan S, Keegan TH, Clarke CA. Disparities in mammographic screening for Asian women in California: a cross-sectional analysis to identify meaningful groups for targeted intervention. BMC Cancer 2007; 7: 201.

15. Cheng LSW, Wong MFF. A survey of the perceived risks of breast cancer in menopausal and postmenopausal women in Hong Kong. GSTF J Nurs Health Care 2016; 4: 57-62.

16. Rosenstock IM. Historical origins of the health belief model. Health Educ Monogr 1974; 2: 328-35.

17. Ersin F, Gözükara F, Polat P, Erçetin G, Bozkurt ME. Determining the health beliefs and breast cancer fear levels of women regarding mammography. Turk J Med Sci 2015; 45: $775-81$.

18. Hacihasanoğlu R, Gözüm S. The effect of training on the knowledge levels and beliefs regarding breast self-examination on women attending a public education centre. Eur $\mathrm{J}$ Oncol Nurs 2008; 12: 58-64.

19. Moballeghi Z, Hatef NE, Najar SH. Health belief about mammography among academician women in Chamran university of Ahvaz. Paper presented at: International Conference on Earth, Environment and Life sciences (EELS2014); 2014 Dec 23-24; Dubai, United Arab Emirates. p. 257.

20. Noroozi A, Jomand T, Tahmasebi R. Determinants of breast self-examination performance among Iranian women: an application of the health belief model. J Cancer Educ 2011; 26: $365-74$.

21. Cohen J. A power primer. Psychol Bull 1992; 112: 155-9.

22. Kwok C, Fethney J, White K. Chinese breast cancer screening beliefs questionnaire: development and psychometric testing with Chinese-Australian women. J Adv Nurs 2010; 66: 191-200

23. Fong DY, Kwok C, White K. Psychometric properties of the Chinese Breast Cancer Screening Beliefs questionnaire. Eur J Oncol Nurs 2012; 16: 505-11.

24. Hosmer DW, Lemeshow S. Applied logistic regression. 2nd ed. New York: John Wiley \& Sons Inc,; 2000.

25. Leung GM, Lam TH, Thach TQ, Hedley AJ. Will screening mammography in the East do more harm than good? Am J Public Health 2002; 92: 1841-6.

26. Chan SS, Chow DM, Loh EK, Wong DC, Cheng KK, Fung WY, et al. Using a community-based outreach program to improve breast health awareness among women in Hong Kong. Public Health Nurs 2007; 24: 265-73.

27. Cheng LSW, Wong MFF. A survey of the perceived lifestyle risks of breast cancer in menopausal and postmenopausal women in Hong Kong. GSTF J Nurs Health Care 2016; 4: 57-62.

28. Wong MFF, Cheng LSW. A survey: knowledge about breast cancer and health beliefs towards screening practice among 
menopausal and postmenopausal women. GSTF J Nurs Health Care 2017; 5: 1-5.

29. Al-Sakkaf KA, Basaleem HO. Breast cancer knowledge, perception and breast self- examination practices among Yemeni women: an application of the health belief model. Asian Pac J Cancer Prev 2016; 17: 1463-7.

30. Okobia MN, Bunker CH, Okonofua FE, Osime U. Knowledge, attitude and practice of Nigerian women towards breast cancer: a cross-sectional study. World J Surg Oncol 2006; 4: 11 .

31. Monninkhof EM, Elias SG, Vlems FA, van der Tweel I,
Schuit AJ, Voskuil DW, et al. Physical activity and breast cancer: a systematic review. Epidemiology 2007; 18: 13757.

32. Secginli S, Nahcivan NO. Factors associated with breast cancer screening behaviours in a sample of Turkish women: a questionnaire survey. Int J Nurs Stud 2006; 43: 16171.

33. Valdez A, Banerjee K, Ackerson L, Fernandez M, Otero-Sabogal R, Somkin CP. Correlates of breast cancer screening among low-income, low-education Latinas. Prev Med 2001; 33: 495-502. 